\title{
Effects of Oxidation of Human Serum Albumin on the Binding of Aripiprazole
}

\author{
Keiki Sakurama, ${ }^{a, \#}$ Koji Nishi, ${ }^{a, \#}$ Victor Tuan Giam Chuang, ${ }^{b}$ Mai Hashimoto, ${ }^{a}$ \\ Keishi Yamasaki, ${ }^{*, a, c}$ and Masaki Otagiri ${ }^{a, c}$ \\ ${ }^{a}$ Faculty of Pharmaceutical Sciences, Sojo Univerity; 4-22-1 Ikeda, Nishi-ku, Kumamoto 860-0082, Japan: ${ }^{b}$ School \\ of Pharmacy, Manash University Malaysia; Bandar Sunway 47500, Selangor, Malaysia: and ${ }^{c}$ DDS Research Institute, \\ Sojo University; 4-22-1 Ikeda, Nishi-ku, Kumamoto 860-0082, Japan. \\ Received March 3, 2020; accepted March 22, 2020
}

Aripiprazole (ARP) is one of antipsychotics and binds to human serum albumin (HSA) with a high affinity. In this study, we investigated the binding characteristics of ARP to oxidized HSA as observed in chronic disease conditions. Oxidized HSAs were prepared using chloramine-T (CT-HSA) or metal-catalyzed oxidation system (MCO-HSA) in vitro, respectively. An increase in the carbonyl content was confirmed in oxidized HSAs. From the results of circular dichroism (CD) and tryptophan fluorescence spectra, no significant structural change of oxidized HSAs was observed. These results indicate that prepared HSAs are mildly oxidized and well reflects the status of HSA during chronic diseases. However, oxidized HSAs were observed to have a significant decrease in binding to ARP. The results of the induced CD spectrum suggested that ARP bound to oxidized HSAs with a similar orientation. These results suggest that oxidation of HSA during chronic disease state significantly affected the microenvironment of the binding site for ARP and binding capacity of HSA to ARP.

Key words albumin; aripiprazole; protein binding; oxidation

\section{INTRODUCTION}

Human serum albumin (HSA) is the most abundant protein in plasma and plays an important role in the regulation of colloidal osmotic pressure and the transport of numerous endogenous compounds such as fatty acids, bilirubin and indoxyl sulfate. ${ }^{1,2)}$ HSA also binds exogenous compounds including drugs. ${ }^{3)}$ In the general circulation, drug molecules exist in equilibrium in the protein bound form (mainly to HSA) or unbound form. Generally, the free, active concentrations of drugs are influenced by the binding of the drugs to HSA. Thus, drug-HSA binding interaction is an important factor in our understanding of the pharmacokinetics and pharmacological effects of various drugs.

Altered HSA binding of drugs has been reported to occur in disease states such as renal failure and diabetics. ${ }^{4,5}$ These diseases are known to generate cellular reactive oxygen species (ROS) that affect the structure and function of various cellular and plasma proteins. Therefore, the binding capacity of HSA may be altered during such diseases due to oxidation by ROS such as hydroxy radical. The understanding of pharmacokinetics and pharmacological activity of drugs in disease state is important for effective patient treatment. In this regard, diagnoses and novel therapy based on drug-HSA interactions are expected to produce a more effective treatment for patients.

Aripiprazole (ARP), 7-(4-(4-(2,3-dichlorophenyl)-1-piperazinyl) buthoxy-3,4-dihydro-2-(1H)-quinolinone, is a novel antipsychotic agent which shows a different pharmacological profile from other antipsychotics. After oral administration of ARP, it is rapidly absorbed from intestinal tract. ${ }^{6)}$ ARP and

\footnotetext{
\# These authors contributed equally to this work.

its main metabolite with pharmacologically activity, dehydroARP, are bound to plasma proteins at therapeutic drug concentrations $(99 \%>)^{7)}$ Very recently, we reported that ARP highly binds to HSA. 8 ,9)

In this study, the binding of ARP by HSA in diseased state was examined using oxidized HSA, in the hope of elucidating the mechanism of drug-HSA interaction. For this purpose, oxidized HSAs by metal-catalyzed oxidation (MCO-HSA) and treatment with chloramine-T (CT-HSA) were prepared as oxidation stress system models in kidney diseases.

\section{MATERIALS AND METHODS}

Materials Fatty acid free HSA were purchased from Sigma-Aldrich Chemicals Co. (St. Louis, U.S.A.). ARP was obtained from Tokyo Chemical Industry Co., Ltd. (Tokyo, Japan). Chloramine-T (CT) and ascorbic acid were purchased from Nacalai Chemical, Ltd. (Kyoto, Japan). Iron(II) chloride tetrahydrate $\left(\mathrm{FeCl}_{2} \cdot 4 \mathrm{H}_{2} \mathrm{O}\right)$ and 2,4-dinitrophenylhydrazine were purchased from FUJIFILM Wako Pure Chemical Corporation (Osaka, Japan). All other chemicals were obtained from commercial sources and were of the highest grade available. About $67 \mathrm{mM}$ sodium phosphate buffer ( $\mathrm{pH}$ 7.4) was used in the equilibrium dialysis and spectroscopic experiments.

Preparation of Oxidized HSA Oxidation with CT was carried out following the method reported by Anraku et al. ${ }^{10)}$ Briefly, $300 \mu \mathrm{M}$ of HSA was reacted with $10 \mathrm{mM}$ of CT in phosphate buffer for $2 \mathrm{~h}$ at $37^{\circ} \mathrm{C}$. Metal-catalyzed oxidation (MCO) of HSA was performed according to the method of Meucci et al. ${ }^{11)}$ Briefly, $300 \mu \mathrm{M}$ of HSA was reacted with $10 \mu \mathrm{M}$ of $\mathrm{FeCl}_{2} \cdot 4 \mathrm{H}_{2} \mathrm{O}$ and $100 \mathrm{mM}$ of ascorbic acid in $50 \mathrm{mM}$ Tris buffer $\left(\mathrm{pH} \mathrm{7.4)}\right.$ for $24 \mathrm{~h}$ at $37^{\circ} \mathrm{C}$. These reactions were stopped by dialysis under low temperature, respectively. After 
dialysis, the samples were freeze-dried and stored at $-20^{\circ} \mathrm{C}$ until use.

Measurement of Carbonyl Contents Carbonyl contents of HSA and oxidized HSAs were determined according to the method of Levine et al. Briefly, the carbonyl groups were derivatized by DNPH and their concentration was calculated using the extinction coefficients of DNPH at $370 \mathrm{~nm}$ $\left(\varepsilon 370 \mathrm{~nm}=22000 \mathrm{M}^{-1} \mathrm{~cm}^{-1}\right)$ using a UV/VIS spectrometer (Ubest-35; JASCO Co., Tokyo, Japan).

Spectroscopy Study Fluorescence spectroscopy measurements were made on a UV/VIS spectrometer (Ubest-35; JASCO Co.). Fluorescent intensities of tryptophan residue were measured at the maximum value around $340 \mathrm{~nm}$ when excited at $285 \mathrm{~nm}$. Circular dichroism (CD) measurements were done using a Jasco model J-720 spectropolarimeter in a cell of $10 \mathrm{~mm}$ pathlength. All measurements were performed at $25^{\circ} \mathrm{C}$.

Equilibrium Dialysis Study Equilibrium dialysis experiments were performed according to our previous reports. ${ }^{8,9}$ Experiments were performed at $25^{\circ} \mathrm{C}$ using $2 \mathrm{~mL}$ Sanko plastic dialysis cells (Fukuoka, Japan) at concentrations of HSA, $40 \mu \mathrm{M}$; and ARP, $20 \mu \mathrm{M}$ to estimate the free fraction of ARP.

HPLC Conditions The HPLC system used in this study consisted of a Hitachi 655 A-11 pump, Hitachi 655 A variable wavelength UV monitor. The eluent was detected at $210 \mathrm{~nm}$. The stationary phase was a YMC-Pack ODS-AM column $(250 \times 4.6 \mathrm{~mm}, 5 \mu \mathrm{m}, 12 \mathrm{~nm}$, YMC Co., Ltd., Kyoto, Japan) maintained at $40{ }^{\circ} \mathrm{C}$ before of $50 \mu \mathrm{L}$ of sample injection. The mobile phase consisted of $50 \mathrm{mM} \mathrm{NaH} \mathrm{PO}_{4} \cdot 2 \mathrm{H}_{2} \mathrm{O}$ [A]-aceto-

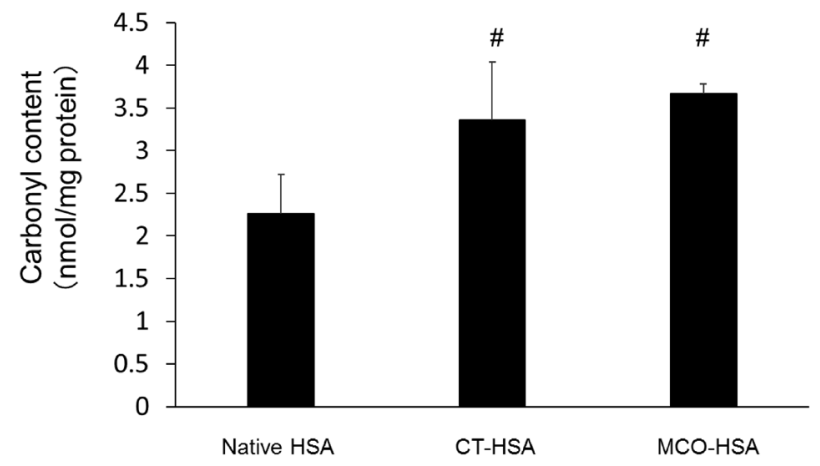

Fig. 1. Carbonyl Content of Oxidized HSAs

Carbonyl contents of modified HSAs were estimated as described in "Materials and Methods." Values are expressed as means \pm standard deviation (S.D.) of three independent experiments. \# $p<0.05$ as compared to native HSA.

A

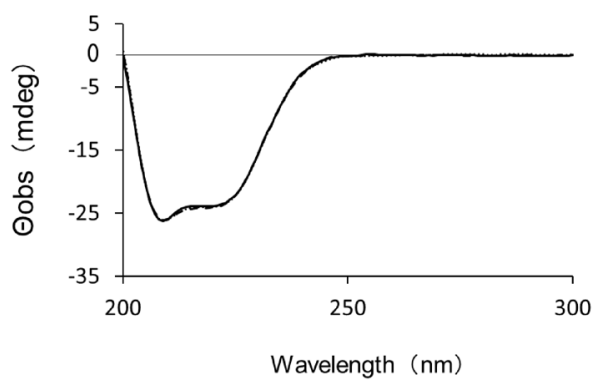

nitrile [B]. The flow rate of the mobile phase was maintained at $1 \mathrm{~mL} / \mathrm{min}$. Gradient step started with $30 \%$ of solvent B and finally reached $70 \%$ in $7 \mathrm{~min}$.

Statistical Analysis Statistical analysis of the data was performed using one-way ANOVA, followed by Tukey multiple comparisons to evaluate differences between the means. For all procedures, GraphPad PRISM ${ }^{\circledR}$ Version 7 (GraphPad Software, Inc., CA, U.S.A.) were used. Differences with $p<0.05$ were considered to be statistically significant.

\section{RESULTS}

Carbonyl Contents of Oxidized HSAs ROS such as hydroxy radical causes carbonylation of arginine, lysine and proline residues in proteins. Thus, carbonylation is used as an indicator of oxidized proteins. Figure 1 shows that both carbonyl contents of CT-HSA and MCO-HSA significantly increased, while there is no significant difference between CTHSA and MCO-HSA.

Structural Properties of Oxidized HSAs Far- and nearUV CD spectra of oxidized HSAs were measured to investigate the effect of oxidation on the secondary and tertiary structure of HSA respectively (Fig. 2). As shown in Figs. 2A and $\mathrm{B}$, both oxidation by $\mathrm{CT}$ and MCO had little effect on the structural properties of HSA. HSA has only one tryptophan residue at 214 (Trp214). To investigate the effect of oxidation on the microenvironment around this residue, the fluorescence intensities of Trp214 of HSAs were measured (Fig. 3). The maximum fluorescent intensities of Trp214 of oxidized HSA (CT-HSA and MCO-HSA) were slightly decreased compared to that of native HSA.

Drug Binding to Oxidized HSAs We examined the binding of ARP to oxidized HSAs. The results obtained were shown in Fig. 4. As can be seen in Fig. 4, the binding affinity of ARP to CT-HSA and MCO-HSA were markedly decreased. In addition, there are no significant difference in the binding of ARP between CT-HSA and MCO-HSA.

Extrinsic Cotton Effects of ARP Bound to Oxidized HSAs Effects of oxidation of HSA on the ARP binding were also examined using CD spectroscopy. The induced CD spectra of protein-ligand complex generally suggest the binding affinity of ligand and the orientation of ligand in the binding pocket. The magnitude of spectra of ARP-modified HSA systems generated slight changes as expected from the results of equilibrium dialysis experiments, while the shapes of spectra were quite similar (Fig. 5).

\section{B}

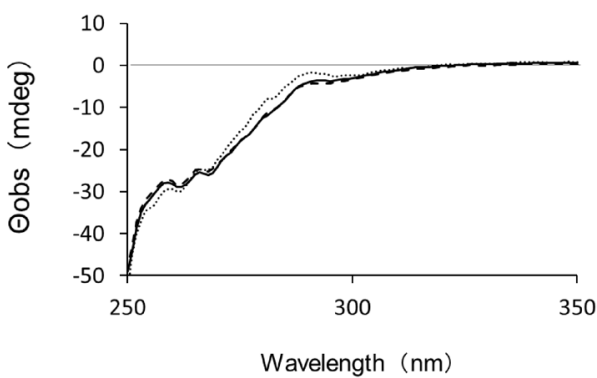

Fig. 2. Far- and Near-UV CD Spectra of Oxidized HSAs

Far (A)- and near (B)-UV CD spectra were measured at 2 and $40 \mu \mathrm{M}$ of HSAs, respectively. Solid, dotted and dashed lines represent native HSA, CT-HSA, and MCOHSA, respectively. The spectra are the average of three determinations. 


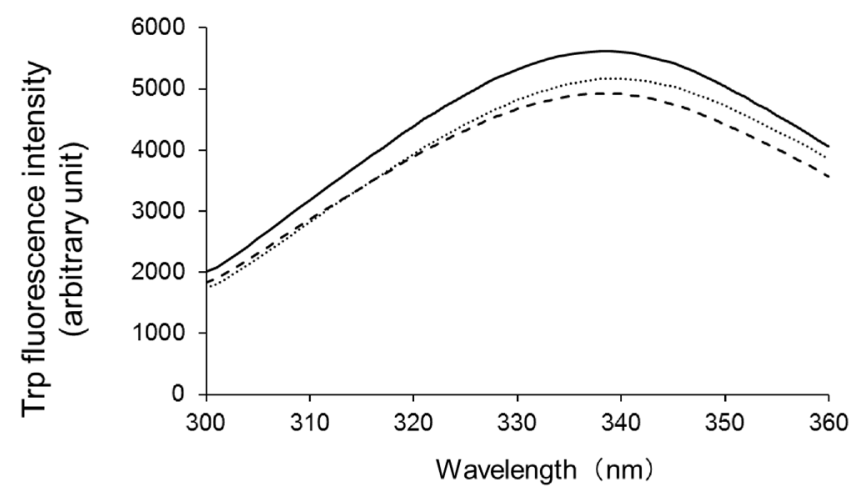

Fig. 3. Intrinsic Fluorescence Spectra of Tryptophan Residue of Oxidized HSAs

Tryptophan fluorescence spectra were measured at $20 \mu \mathrm{M}$ of HSAs. Solid, dotted and dashed lines represent native HSA, CT-HSA and MCO-HSA, respectively. The spectra are the average of three determinations.

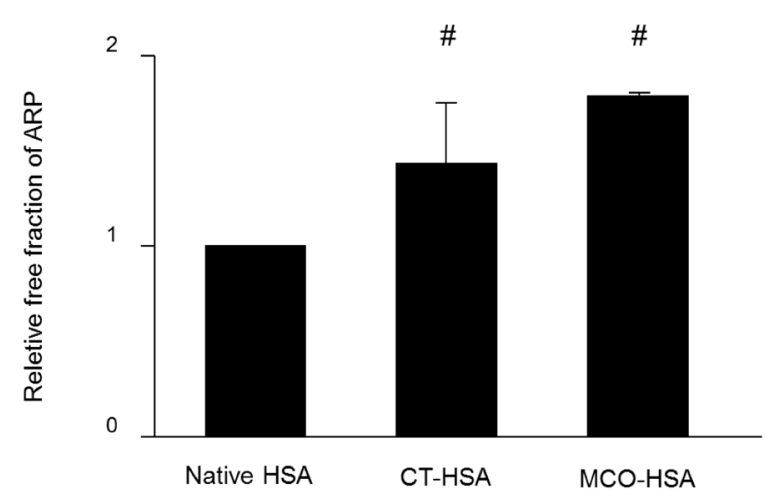

Fig. 4. Relative Free Fraction of ARP to Oxidized HSAs

Free fractions of ARP were estimated by equilibrium dialysis. The concentrations of ARP and HSAs were 20 and $40 \mu \mathrm{M}$, respectively. Values are expressed means \pm S.D. of three independent experiments. ${ }^{\#} p<0.05$ as compared to native HSA.

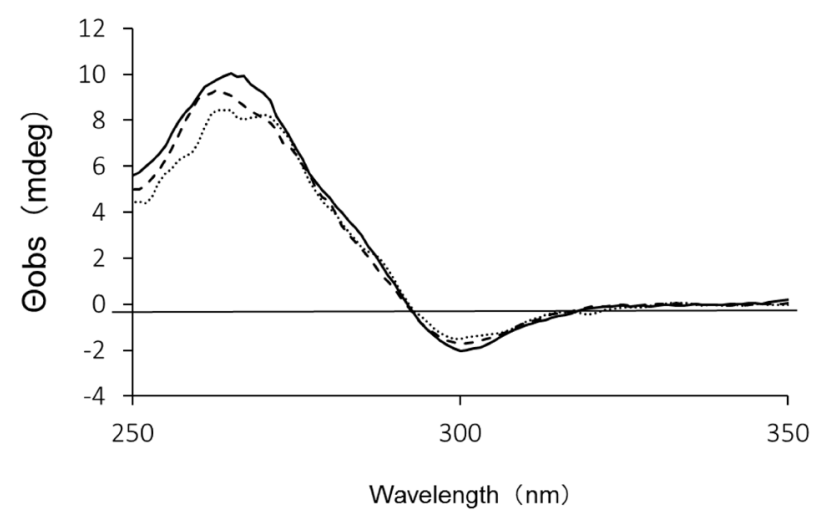

Fig. 5. Induced CD Spectra of ARP-HSAs

The concentration of ARP and HSAs were 20 and $40 \mu \mathrm{M}$, respectively. The spectra are the average of three determinations. Solid, dotted and dashed lines represent native HSA, CT-HSA and MCO-HSA, respectively.

\section{DISCUSSION}

In several disease states, altered concentrations and structural changes of HSA, and accumulated endogenous substances have been reported to be able to affect drug binding to HSA. ${ }^{4,5)}$ Decreased drug binding to HSA is known to occur especially in renal, liver and diabetic diseases. In this study, oxidized HSAs (CT-HSA and MCO-HSA) were prepared as the oxidized HSA models because these models were reported to well reflect the modified HSAs observed during chronic disease such as renal diseases. ${ }^{7,10)}$

The carbonyl contents of oxidized HSAs increased slightly but significantly for CT-HSA and MCO-HSA. This result suggests only slight extent of oxidation for HSAs prepared in this study. The result of far- and near-UV CD spectra indicated little change of structural properties of CT-HSA and MCOHSA (Fig. 2). The fluorescent spectra derived from Trp214 was decreased by the oxidations of HSA. This implies that the microenvironment around Trp214 residue has been affected by oxidation.

Oxidation of HSA, CT-HSA and MCO-HSA, caused lowering of ARP binding. According to the literatures, Cys and Met residues could have been oxidized in the preparation of CT-and MCO-HSAs. ${ }^{12,13)}$ In addition, Lys and Arg residues might be oxidized too. Recently, we reported that ARP binds to site II in subdomain IIIA of HSA. Arg410 residue, the key amino acid residue involved in site II ligand binding, was reported to be important in the oxidative stress of HSA. ${ }^{14)} \mathrm{We}$ have previously reported reduced site II ligand binding to CTand MCO-HSAs. In addition, the catalytic activity towards $p$-nitrophenyl acetate was affected in both of CT-HSA and MCO-HSA. This activity of HSA depends on the proximity of Arg410 and Tyr411. ${ }^{10,15)}$ Therefore, the observed decreases in ARP binding to oxidized HSAs may be due to oxidation of Arg and Lys residues in the entrance of site II. Generally, the decrease in the fluorescence intensity of a Trp residue tends to correlate with the drug binding ability, but the details have not been revealed. The results suggest that oxidation of Arg and Lys at site II may have indirectly affected the environment around Trp214.

The findings presented here provide fundamental and valuable information concerning our knowledge of alterations in ARP binding in certain diseased states.

Conflict of Interest The authors declare no conflict of interest.

\section{REFERENCES}

1) Peters T Jr. All about albumin: biochemistry, genetics and medical application. Academic Press Inc., San Diego (1996).

2) Otagiri M, Chuang VT. Albumin in medicine: pathological and clinical applications. Springer Singapore, (2016).

3) Kragh-Hansen U, Chuang VT, Otagiri M. Practical aspects of the ligand binding and enzymatic properties. Biol. Pharm. Bull., 25, 695-704 (2002).

4) Gulyassy PF, Depner TA. Impaired binding of drugs and endogenous ligands in renal diseases. Am. J. Kidney Dis., 2, 578-601 (1983).

5) Yamasaki K, Chuang VT, Maruyama T, Otagiri M. Albumin-drug interaction and its clinical implication. Biochim. Biophys. Acta, 1830, 5435-5443 (2013).

6) Burris KD, Molski TF, Xu C, Ryan E, Tottori K, Kikuchi T, Yocca FD, Molinoff PB. Aripiprazole, a novel antipsychotic, is a highaffinity partial agonist at human dopamine D2 receptors. J. Pharmacol. Exp. Ther., 302, 381-389 (2002).

7) Swainston Harrison T, Perry CM. Aripiprazole: a review of its use in schizophrenia and schizoaffective disorder. Drugs, 64, 1715-1736 (2004).

8) Sakurama K, Kawai A, Tuan Giam Chuang V, Kanamori Y, Osa 
M, Taguchi K, Seo H, Maruyama T, Imoto S, Yamasaki K, Otagiri M. Analysis of the binding aripiprazole to human serum albumin: the importance of a chloro group in the chemical structure. $A C S$ Omega, 3, 13790-13797 (2018).

9) Sakurama K, Nishi K, Imoto S, Hashimoto M, Komatsu T, Morita Y, Taguchi K, Otagiri M, Yamasaki K. Further evidences regarding the important role of chlorine atoms of aripiprazole on binding to site II area of human albumin. J. Pharm. Sci., 108, 1890-1895 (2019).

10) $\overline{\text { Anraku }}$ M, Yamasaki K, Maruyama $T$, Kragh-Hansen U, Otagiri M. Effect of oxidative stress on the structure and function of human serum albumin. Pharm. Res., 18, 632-639 (2001).

11) Meucci E, Mordente A, Martorana GE. Metal-catalyzed oxidation of human serum albumin: conformational and functional changes. Implications in protein aging. J. Biol. Chem., 266, 4692-4699 (1991).
12) Salavej P, Spalteholz H, Arnhold J. Modification of amino acid residues in human serum albumin by myeloperoxidase. Free Radic. Biol. Med., 40, 516-525 (2006).

13) Iwao Y, Ishima Y, Yamada J, Noguchi T, Kragh-Hansen U, Mera K, Honda D, Suenaga A, Maruyama T, Otagiri M. Quantitative evaluation of the role of cysteine and methionine residues in the antioxidative activity of human serum albumin using recombinant mutants. IUBMB Life, 64, 450-454 (2012).

14) Iwao Y, Anraku M, Yamasaki K, Kragh-Hansen U, Kawai K, Maruyama T, Otagiri M. Oxidation of Arg-410 promotes the elimination of human serum albumin. Biochim. Biophys. Acta, 1764, 743-749 (2006)

15) Otagiri M. A molecular functional study on the interaction of drugs with plasma protein. Drug Metab. Pharmacokinet., 20, 309-323 (2005). 